\title{
SYNERGY OF A NOVEL ANTIBIOTIC ADJUVANT ENTITY AGAINST MULTI DRUG RESISTANT ENTEROBACTERIACEAE
}

\author{
Manu Chaudhary and Anurag Payasi \\ Department of Cell Culture and Molecular Biology, \\ Venus Medicine Research Centre, Baddi, H.P., 173205, India
}

Received 2013-10-10, Revised 2013-11-01; Accepted 2013-11-01

\begin{abstract}
In the present investigation, we investigated the in vitro interaction of ceftriaxone plus sulbactam with disodium edetate, a Non Antibiotic Adjuvant (NAA) against selected clinical isolates and in vitro susceptibility studies were also performed. The isolates were tested against a range of ratios of ceftriaxone and sulbactam using a microdilution checkerboard method. Having determined the appropriate ratios of ceftriaxone plus sulbactam, effect of various concentration of disodium edetate were also studied using the microdilution checkerboard method. All the results were analysed with the Fractional Inhibitory Concentration (FIC) indices. Susceptibility studies were carried out according to the Clinical and Laboratory Standards Institute (CLSI) methods. Results of this study demonstrated that 2:1 ratio of ceftriaxone and sulbactam was the more synergistic with FIC index values $0.4281,0.4023,0.4124$ and 0.4325 for E. coli, A. baumannii, $P$. aeruginosa and $K$. pneumoniae. The synergicity of ceftriaxone and sulbactam was enhanced significantly with increasing concentration of disodium edetate and produced the lowest FIC index $(<0.2)$ at $10 \mathrm{mM}$ of disodium edetate in all positive controls as well as clinical isolates. Further, the synergy between ceftriaxone plus sulbactam with disodium edetate (Elores) was confirmed by broth dilution, time kill curve and agar diffusion methods. In broth dilution method, Elores (ceftriaxone+sulbactam+disodium edetate) produced 4 to 5 fold lower MIC when compared with ceftriaxone plus sulbactam. Approximately $10^{4} \log$ of killing reduction was observed with synergistic ratio of Elores in time kill curve study. This study suggest that Elores could be an alternative regimen in combating antibiotic resistance among multi drug resistant Enterobacteriaceae.
\end{abstract}

Keywords: Clinical Isolates, Enterobacteriaceae, FIC Index, Synergy

\section{INTRODUCTION}

Increasing resistance to 3rd generation cephalosporins particlularly due to extended spectrum beta lactamase production has become a major concern especially among Enterobacteriaceae that cause nosocomial infections (Rawat and Nair, 2010). Approximately $20 \%$ of Klebsiella pnenumoniae infections and $31 \%$ of Enterobacter species infections in intensive care unit in the United States now involve strains not susceptible to 3rd-generation cephalosporins.
Salmonella species also getting resistant to expandedspectrum cephalosporins have been reported in several countries, including Argentina, Turkey, Algeria, Saudi Arabia, Greece, Tunisia and France (Dutil et al., 2010). In addition, most of the bacteria, responsible for community-acquired infections have developed resistance to many antibacterial agents particularly betalactams which are being used in over $50 \%$ of all systemic antibiotics (Acevedo et al., 2009). Besides that, several evidences pointed towards the development of resistance to extended-spectrum cephalosporins in bacteria isolated Corresponding Author: Anurag Payasi, Department of Cell Culture and Molecular Biology, Venus Medicine Research Centre, Baddi,
H.P., 173205, India 
from patients with nosocomial infections (Rawat and Nair, 2010). An increasing number of reports have indicated the steady rise in resistance for ceftriaxone (Unemo et al., 2010; Ohnishi et al., 2011). In addition, aminoglycosides, fluroquinolones and carbapenems have usually been used for the treatment of infections caused by Enterobacteriaceae (Howard et al., 2012; Tam et al., 2010). However, in recent years, these organisms have been reported to be resistant to these commonly used antimicrobial agents worldwide (Chaudhary and Payasi; 2012; Memish et al., 2012; Muthusamy and Boppe, 2012). Acquisition or expression of Metallo- $\beta$-Lactamases (MBLs), Extended-Spectrum $\beta$-Lactamases (ESBLs), decreased permeability, overexpression of efflux pump are thought to be the main factors contributing to antibiotic resistance development (Chaudhary and Payasi, 2012; Zavascki et al., 2010; Karthika et al., 2009).

The treatment of infections caused by these microorganisms impose a major challenge to health care system due to failure of monotherapy and lacking of effective regimens. Combination antibiotics have been used frequently in clinical practice, but not all of them work synergistically.

Considering the above background, a team of Venus Medicine Research Centre (VMRC), India has developed a novel Antibiotic Adjuvant Entity (AAE) combination of ceftriaxone with a beta lactamase inhibitor sulbactam and a nonantibiotic adjuvant disodium edetate naming Elores. This AAE can be used successfully for the therapy of infections caused by resistant organisms.

The checkerboard titration method was used to test synergy of various ratios of ceftriaxone and sulbactam against selected clinical isolates and results have been presented in term of the Fractional Inhibitory Concentration Index (FICI). The present study was aimed to differentiate the performance of product on Extended-Spectrum Beta-Lactamase (ESBL), MetalloBeta-Lactamase (MBL) and efflux positive strains. We investigated the in vitro interactions between ceftriaxone and sulbactam with a Non Antibiotic Adjuvant (NAA) disodium edetate using a checkerboard method. Further, the effect of different concentrations of disodium edetate on the double combination of ceftriaxone and sulbactam was studied in detail to determine whether the apparent synergistic interaction between ceftriaxone and sulbactam is enhanced or diminished by the addition of disodium edetate. Furthermore, we studied the in vitro susceptibilities of these isolates to combinations of ceftriaxone and sulbactam and disodium edetate by the use of broth dilution, disk diffusion and time-kill methods.

\section{MATERIALS AND METHODS}

\subsection{Clinical Isolates Collection and Their Identification}

A total of 140 clinical isolates 35 of each E. coli, $K$. pneumoniae, A. baumannii and $P$. aeruginosa were included in the study. The re-identification of clinical isolates were done according to standard microbiological procedures (Khan et al., 2011). Escherichia coli ATCC-35218, K. pneumoniae ATCC BAA-2146 and P. aeruginosa K1455 were included in the study as positive controls. The clinical isolates were obtained from clinical isolate bank of Venus Medicine Research Centre, Baddi and Baba Farid Medical College, Faridkot, Punjab, India, where clinical isolates are preserved. Each of these bacterial cultures were grown and adjusted to 0.5 MacFarland standard.

\subsection{ESBL and MBL Characterization}

All these isolates were subjected to ESBL and MBL characterization as previously described (CLSI, 2011; Yong et al., 2002).

\subsection{Efflux Pump Characterization}

All the isolates positive with ESBL and MBL were further subjected for identification of AcrAB-tolC, mexAB-oprM and AdeABC efflux pumps using the methods described earlier (Chaudhary et al., 2012a; Chaudhary and Payasi, 2012; Lopes and Amyes, 2013).

\subsection{Fractional Inhibitory Concentration (FIC) Study}

In vitro drug interaction was determined by the checkerboard method as described by Wijayanti et al. (2010) and results were analyzed with the FIC indices. For each ratio, a two-dimensional checkerboard with twofold dilutions was used for the study. Growth control wells containing medium were included in each plate. Each test was performed in triplicate. The concentration of antibiotics needed to inhibit growth was recorded. The following formula was used to calculate FIC:

$$
\text { FIC }=\frac{\text { MIC of drug in combination }}{\text { MIC of drug alone }}
$$

The FIC index ( $\sum$ FIC) calculated as the sum of each FIC, was interpreted as follows: Synergy is defined as an FIC index of $\leq 0.5$. Antagonism is defined as an FICindex 
of $\geq 2$. An indifferent/additive effect is defined as an FIC index of $>0.5$ to 2 or a micro dilution decrease of 1 dilution in the MIC of the one or the other drug or no change in the MIC of either of the drugs.

\subsection{Effect of Non-Antibiotic Adjuvant (NAA) on Double Combinations}

Effect of NAA on $\sum$ FIC of double combinations, ceftriaxone plus sulbactam was also conduced using checkerboard method (Wijayanti et al., 2010) in the absence and presence of increasing concentration of disodium edetate in all positive controls as well as clinical isolates.

\subsection{Determination of Minimum Inhibitory Concentration (MIC)}

MICs were determined by broth dilution method following the guidelines of the CLSI (2011) using cation-adjusted Mueller-Hinton Broth (MHB) [HiMedia, India]. MIC was defined as the lowest concentration of antibiotic that completely inhibited the growth of the organism as detected with the naked eye.

\subsection{Determination of Antimicrobial Susceptibility Test (AST)}

AST was determined according to the cup plate method described by Chaudhary et al. (2012b). The cups were made in the agar plate using a sterile cork borer (6.5 mm). Then, $30 \mu \mathrm{L}$ of the drug preparation Elores (ceftriaxone+sulbactam+disodium edetate $(30: 15 \mu \mathrm{g})$, ceftriaxone+sulbactam $(30: 15 \mu \mathrm{g})$ and ceftriaxone $(30 \mu \mathrm{g})$ were placed into the wells using a micro-pipette and allowed the plates to incubate at $37^{\circ} \mathrm{C}$ for $18 \mathrm{~h}$ in the upright position. After incubation the zone of inhibition around the wells was measured in $\mathrm{mm}$ (millimeter), averaged and the mean values were recorded.

\subsection{Determination of Time Kill Curve (TKC)}

TKC study was performed according to CLSI (2011) guidelines. Twice the MIC of ceftriaxone, ceftriaxone+sulbactam and Elores (ceftriaxone+sulbactam+disodium edetate) was used for this study. For TKC study, two randomly selected clinical isolate of each E. coli, K. pneumoniae, P. aeruginosa and A. baumannii and all positive controls were used. Overnight grown bacterial suspension was diluted to approximately $10^{6}$ to $10^{7} \mathrm{cfu} \mathrm{mL}^{-1}$ in MHB containing antibiotics or no antibiotics. The samples were removed at 2, 4, 6, 8, 10 and $12 \mathrm{~h}$ and were diluted and plated on MHA. The agar plates were incubated at $37^{\circ} \mathrm{C}$ for $24 \mathrm{~h}$ and colony forming unit (cfu) were counted.

\section{RESULTS}

\subsection{Clinical Isolate Identification and Characterization}

All of the clinical isolates obtained from isolate banks were identified as A. baumannii, E. coli, P. aeruginosa and $K$. pneumoniae based on their morphological and biochemical characterization. Out of the 140 isolates, 60 were found to be ESBL positive (A. baumannii 11, E. coli $15, P$. aeruginosa $16, K$. pneumoniae 18$)$ and 47 were MBL posiitve (A. baumannii 5, E. coli 12, P. aeruginosa $9, K$. pneumoniae 11). Out of these ESBL positive isolates, 27 isolates were efflux positive $[A$. baumannii 7 (AdeABC positive), E. coli 8 (AcrABC-tolC positive), $P$. aeruginosa 5 (mexABoprM positive), $K$. pneumoniae 7(AcrAB-tolC positive)]. Similarly among MBL positive isolates, 14 were efflux positive $[A$. baumannii 3 (AdeABC positive), E. coli 5 (AcrABC-tolC positive), $P$. aeruginosa 3 (mexABoprM positive), $K$. pneumoniae 4 (AcrAB-tolC positive)].

\subsection{FIC Study}

Figure 1 summarizes the results of the FIC index analysis of the various ratios of ceftriaxone and sulbactam tested against E. coli, A. baumannii, $P$. aeruginosa and $K$. pneumoniae. The results demonstrated that $2: 1$ ratio of ceftriaxone and sulbactam was the most synergistic. Further increasing the ratio of either ceftriaxone or sulbactam synergistic activity was either lost or no further potentiation was observed. This study was conducted in all selected clinical isolates as well as positive controls and synergistic activity was noted at 2:1 ratio of ceftriaxone and sulbactam. The results of one clinical isolate of each E. coli, $A$. baumannii, $P$. aeruginosa and $K$. pneumoniae positive with both MBL and efflux is presented here only.

\subsection{Effect of NAA on Double Combinations}

Effect of NAA, disodium edetate on FIC indices of double combinations ceftriaxone plus sulbactam in the absence and presence of increasing concentration of disodium edetate was done in all 140 isolates of E. coli, A. baumannii, P. aeruginosa and K. pneumoniae and positive controls. Results of this study showed that $\sum$ FIC decreased with increasing concentration of disodium edetate and maximum decrease was found $10 \mathrm{mM}$ of disodium edetate. Further increasing the concentration of disodium edetate $\sum$ FIC remained constant. The FIC analysis for four selected clinical isolates which were used for FIC study are presented in Fig. 2. 

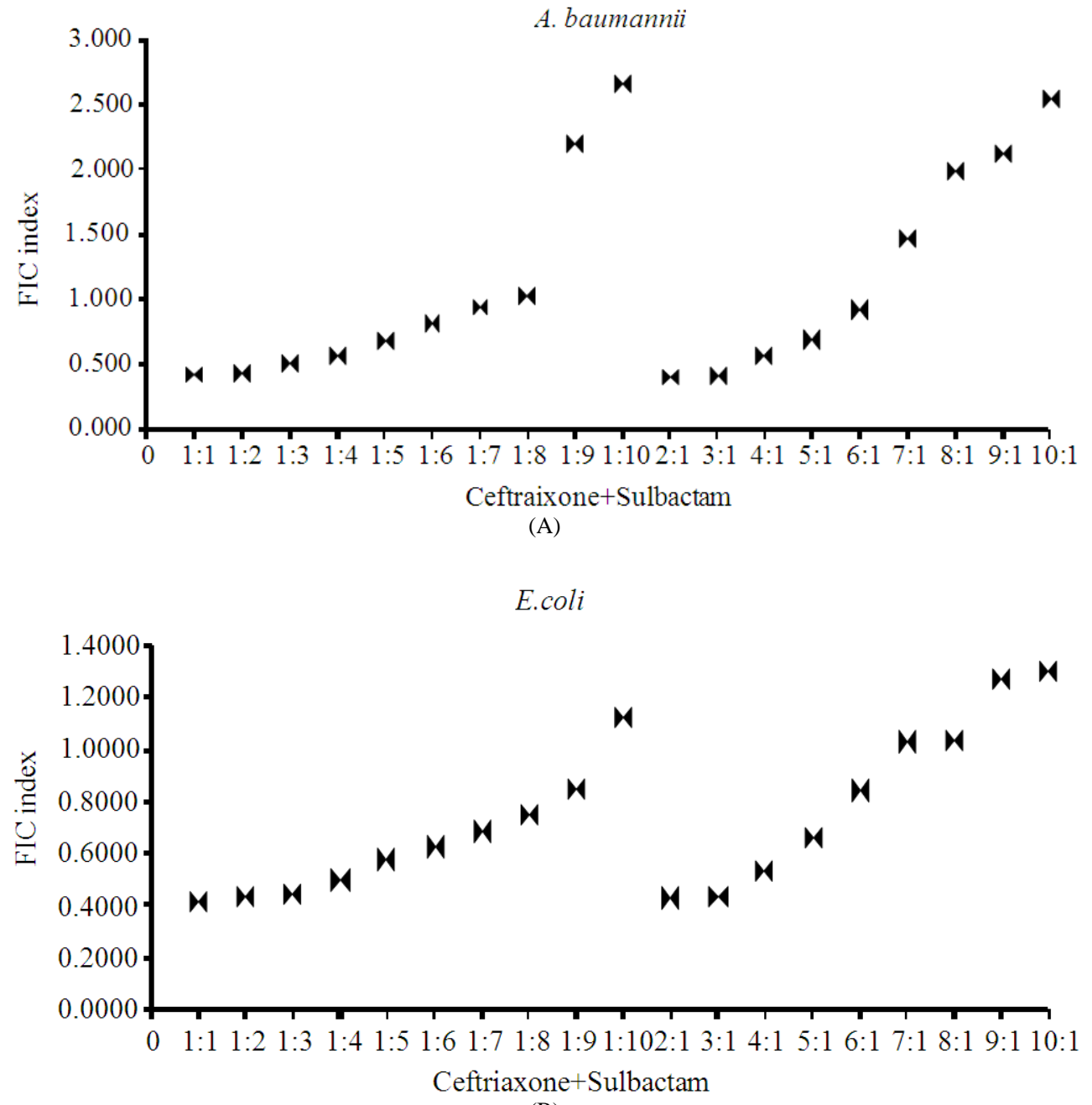

(B)

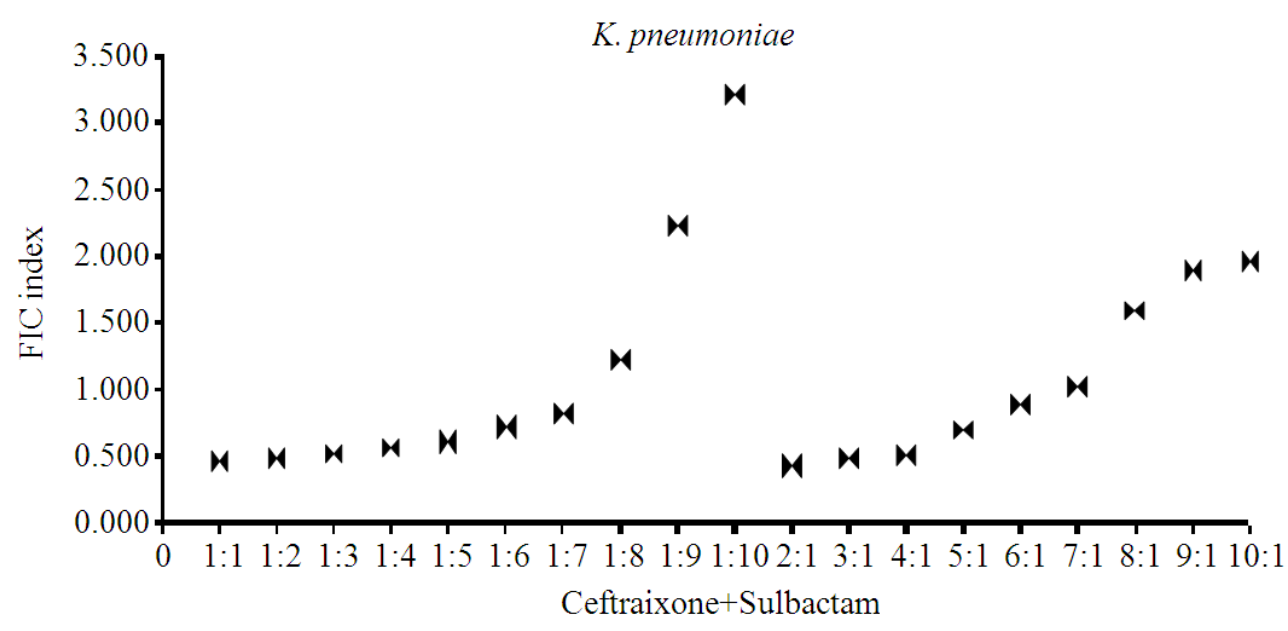

(C) 


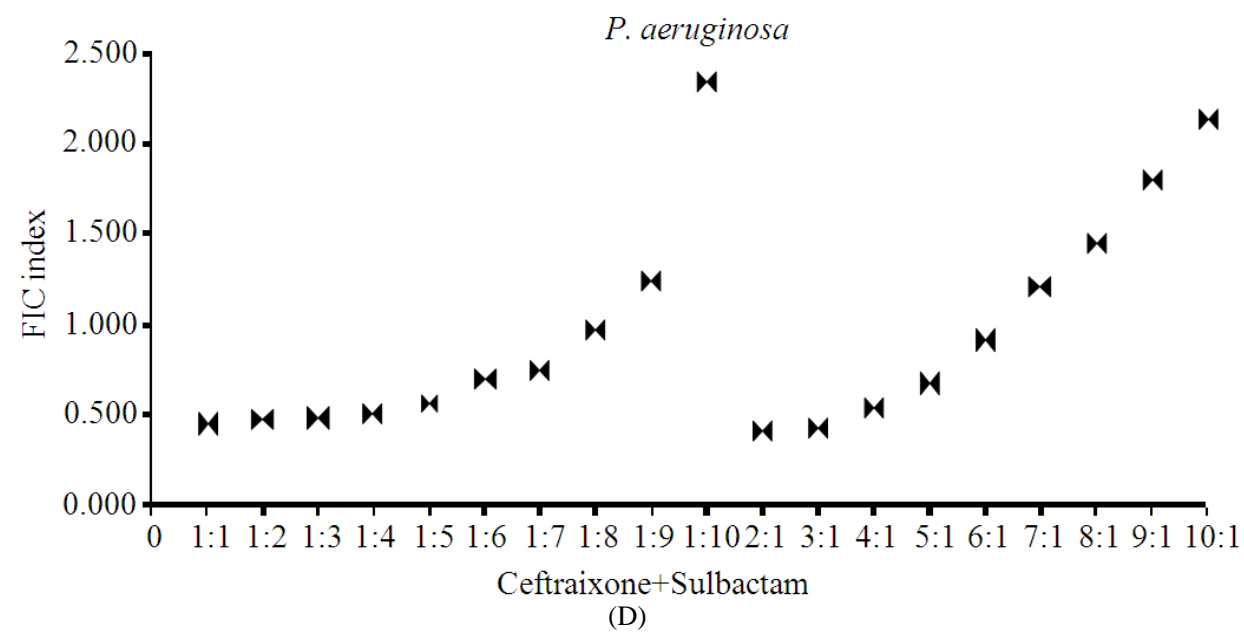

Fig. 1. FIC indices at various ratios of ceftriaxone and sulbactam against MBL positive clinical isolates with efflux. FIC indices of ceftriaxone and sulbactam in the presence of increasing concentration of sulbactam (1:1 to $1: 10)$ and then ceftriaxone (2:1 to 10:1). (A) A. baumannii (B) E. coli (C) K. pneumoniae (D) P. aeruginosa. FIC index synergistic when value is $\leq 0$

\section{A. baumannii}

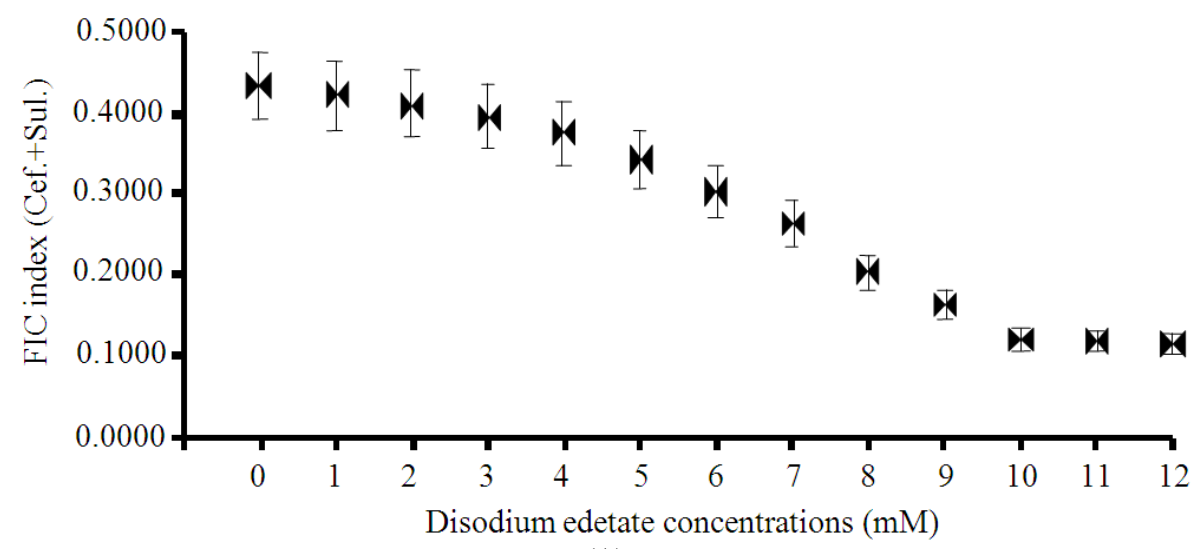

(A)

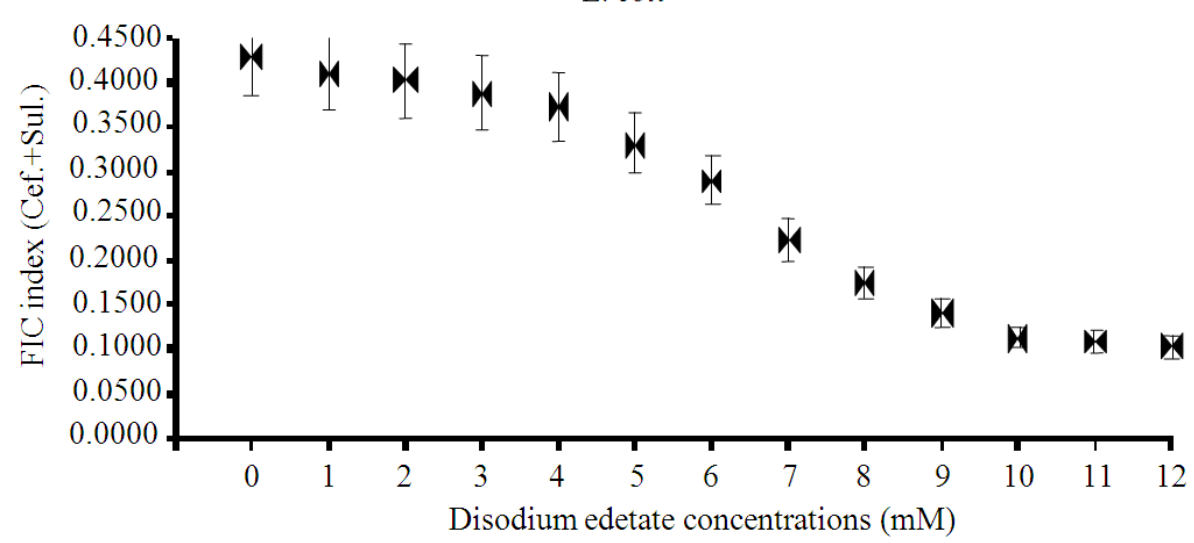

(B) 

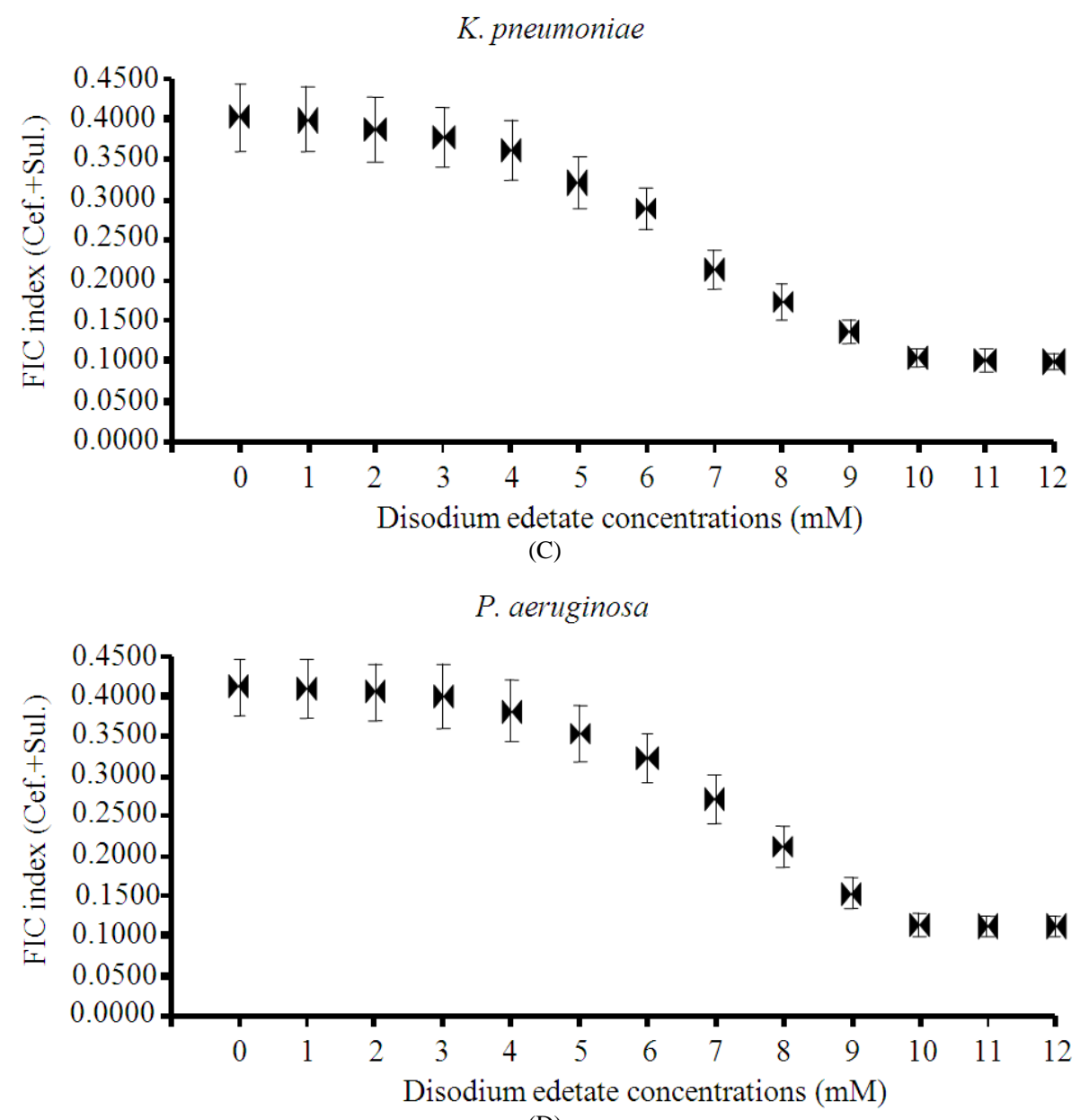

(D)

Fig. 2. Effect of disodium edetate on the combination of ceftriaxone and sulbactam against MBL positive clinical isolates with efflux. FIC index of ceftriaxone and sulbactam in the presence of increasing concentration of disodium edetate (A) A. baumannii (B) E. coli (C) K. pneumoniae (D) P. aeruginosa. FIC index synergistic when value is $\leq 0.5$

From $\sum$ FIC analysis of all clinical isolates, FICI $_{\min }$ and FICI $_{\max }$ were calculated and results are presented in Fig. 3. The FICI $_{\text {min }}$ and FICI $_{\max }$ were significantly lower equal to less than 0.5 , which indicates the presence of synergistic interactions among the three combinations.

\subsection{MIC}

Synergism between ceftriaxone and sulbactam along with NAA was also performed by a broth dilution method against selected clinical isolates and positive isolates. The MICs for positive controls ranged 2-4 $\mu \mathrm{g} \mathrm{mL}{ }^{-1}$ for ceftriaxone+sulbactam+disodium edetate (Elores), whereas it was ranged between 512->1024 and 256-512 for ceftriaxone and ceftriaxone+sulbactam, respectively (Table 1). MICs for Elores were 4-32 $\mu \mathrm{g} \mathrm{mL}^{-1}$ for clinical isolates of A. baumannii and 4-16 $\mu \mathrm{g} \mathrm{mL}^{-1}$ for each of E. coli, K. pneumoniae and P. aeruginosa positive with ESBL. MICs for Elores to MBL positive isolates of $A$. baumannii and $E$. coli were 2-16 and 1-8 $\mu \mathrm{g} \mathrm{mL}^{-1}$, respectively whereas it was ranged 2-8 $\mu \mathrm{g} \mathrm{mL}^{-1}$ for $K$. pneumoniae and $P$. aeruginosa isolates. Similarly, MICs for Elores against efflux positive isolates were 2-16 $\mu \mathrm{g} \mathrm{mL}^{-1}$ for each of A. baumannii, $K$. pneumoniae and $P$. aeruginosa and $1-8 \mu \mathrm{g} \mathrm{mL}^{-1}$ for $E$. coli. Contrary to this, ceftriaxone MICs were $>1024$ to all the isolates except $E$. coli and $K$. pneumoniae positive with efflux. Ceftriaxone+sulbactam demonstrated MICs values 4-6 fold higher than Elores in all isolates (Table 1). MIC studies were also conducted using other ratios (1:1, 1:2, 3:1 and 4:1) of ceftriaxone plus sulbactam but significant results were obtained only with $2: 1$ ratio. 


\subsection{AST}

Synergism of ceftriaxone and sulbactam against $A$. baumannii, E. coli, K. pneumoniae and $P$. aeruginosa were also demonstrated by a cup-plate agar diffusion method. For positive controls of E. coli, K. pneumoniae and $P$. aeruginosa inoculated onto a MHA plate containing Elores produced a $\geq 5 \mathrm{~mm}$ enhanced zone of inhibition $25.78 \pm 1.4,26.24 \pm 1.8$ and $25.53 \pm 1.6 \mathrm{~mm}$, respectively compared to ceftriaxone alone and ceftriaxone plus sulbactam, indicating enhanced synergistic activity between the ceftriaxone and sulbactam in presence of non antibiotic adjuvant disodium edetate (Table 1). Similarly for clinical isolates of $A$. baumannii, E. coli, K. pneumoniae and $P$. aeruginosa positive with ESBL, MBL and efflux, ceftriaxone plus sulbactam with disodium edetate combination (Elores) produced a greater zone of inhibition $\geq 5 \mathrm{~mm}$ when compared with other two groups (Table 1). AST studies were also conducted using other ratios $(1: 1,1: 2,3: 1$ and 4:1) of ceftriaxone and sulbactam but did not show significant synergy (data not shown).

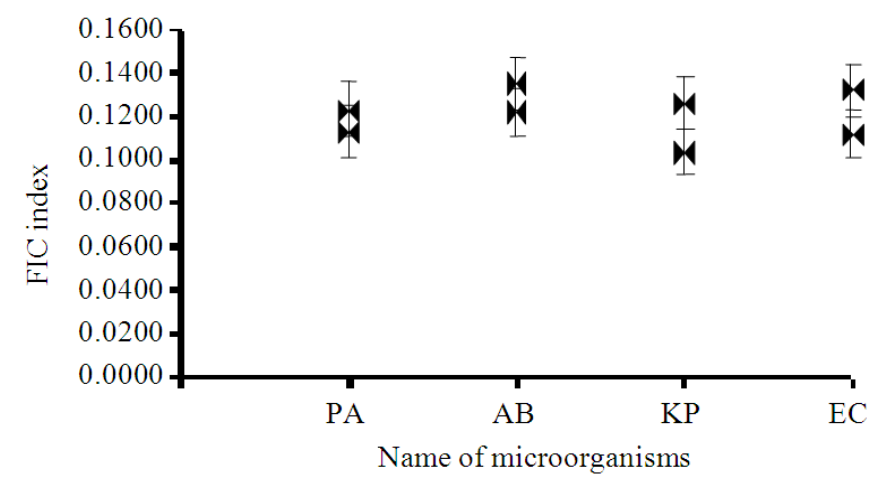

Fig. 3. FICmin and FICmax of the combination of ceftriaxone, disodium edetate and vancomycin against clinical isolates

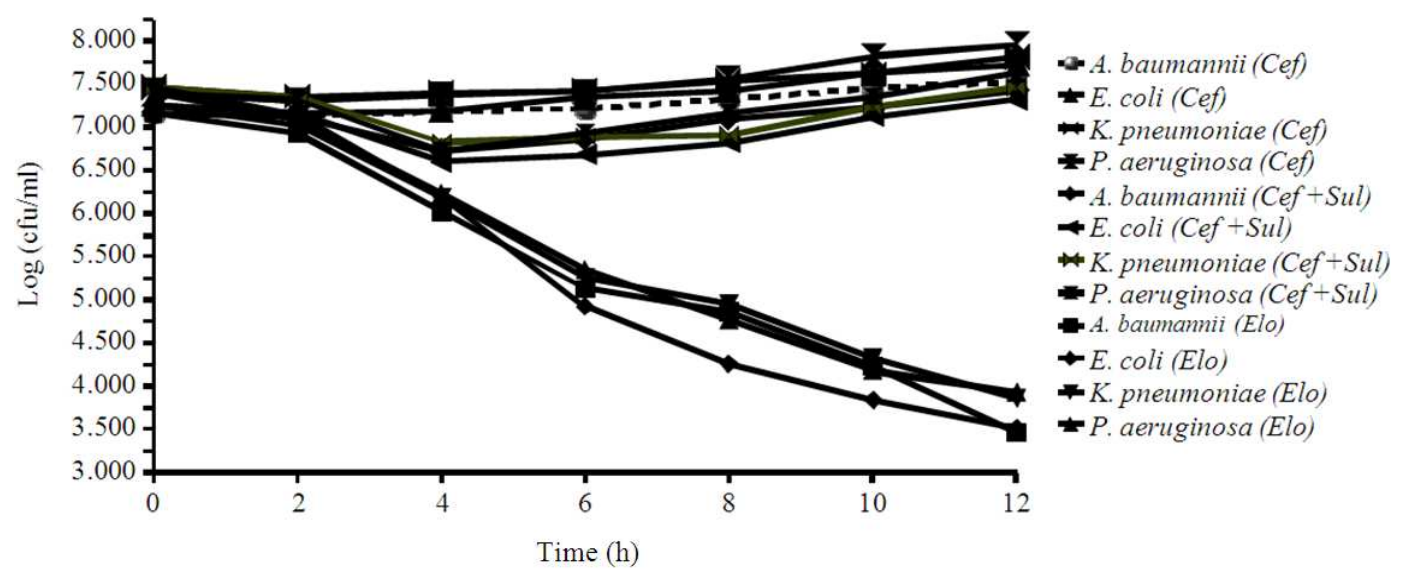

Fig. 4. Time-kill curves for E. coli, K. pneumoniae, P. aeruginosa and A. baumannii isolates positive with both MBL and efflux pump 
Table 1. AST and MIC of antibacterial agents against selected clinical isolates

\begin{tabular}{|c|c|c|c|c|c|c|c|}
\hline \multirow[b]{2}{*}{ Strain no. } & & \multicolumn{3}{|c|}{ Zone of Inhibition (mm) } & \multicolumn{3}{|c|}{ MIC $(\mu \mathrm{g} / \mathrm{mL})$} \\
\hline & & Ceftriaxon & $\begin{array}{l}\text { Ceftriaxone } \\
\text { sulbactam }\end{array}$ & $\begin{array}{c}\text { Ceftriaxone } \\
+\quad+\text { sulbactam } \\
\text { disodium edetate }\end{array}$ & Ceftriaxone & $\begin{array}{c}\text { Ceftriaxone } \\
+ \text { sulbactam }\end{array}$ & $\begin{array}{c}\text { Ceftriaxone } \\
\text { +sulbactam } \\
\text { disodium edetate }\end{array}$ \\
\hline E.coli ATCC-35218 & ESBL positive & $\mathrm{NZ}$ & $20.37 \pm 1.2$ & $25.78 \pm 1.4$ & $>1024$ & 512 & 4 \\
\hline K. pneumonia АТCC ВАА- 2146 & MBL positive & $\mathrm{NZ}$ & $13.26 \pm 1.2$ & $26.24 \pm 1.8$ & $>1024$ & 512 & 2 \\
\hline P. aeruginosaMexA- MexB-OprM K1455 & Efflux positive & $11.25 \pm$ & $17.38 \pm 1.5$ & $25.53 \pm 1.6$ & 512 & 256 & 2 \\
\hline \multirow[t]{3}{*}{ A. baumannii } & ESBL & $8.26 \pm 1.6$ & $10.43 \pm 1.1$ & $24.13 \pm 1.6$ & $>1024$ & $16-256$ & $4-32$ \\
\hline & MBL & $7.13 \pm 1.9$ & $11.56 \pm 1.3$ & $26.43 \pm 1.5$ & $>1024$ & $128-1024$ & $2-16$ \\
\hline & Efflux & $8.46 \pm 1.1$ & $11.84 \pm 1.5$ & $25.57 \pm 1.8$ & $>1024$ & $32-512$ & $2-16$ \\
\hline \multirow[t]{3}{*}{ E. coli } & ESBL & $8.21 \pm 1.4$ & $10.43 \pm 1.3$ & $26.44 \pm 1.1$ & $>1024$ & $64-512$ & $4-16$ \\
\hline & MBL & $7.26 \pm 1.1$ & $9.65 \pm 1.7$ & $27.23 \pm 1.3$ & $>1024$ & $128-1024$ & $1-8$ \\
\hline & Efflux & $8.43 \pm 1.5$ & $10.13 \pm 1.4$ & $25.38 \pm 1.5$ & $512-1024$ & $64-512$ & $1-8$ \\
\hline \multirow{3}{*}{ K. pneumonaie } & ESBL & $8.58 \pm 1.7$ & $9.49 \pm 1.4$ & $24.32 \pm 1.2$ & $>1024$ & $32-256$ & $4-16$ \\
\hline & MBL & $6.96 \pm 1.3$ & $9.57 \pm 1.1$ & $27.32 \pm 1.4$ & $>1024$ & $128-1024$ & $2-8$ \\
\hline & Efflux & $7.63 \pm 1.2$ & $10.47 \pm 1.2$ & $25.23 \pm 1.4$ & $128-1024$ & $64-512$ & $2-16$ \\
\hline \multirow[t]{3}{*}{ P. aeruginosa } & ESBL & $7.46 \pm 1.1$ & $9.88 \pm 1.6$ & $23.23 \pm 1.3$ & $>1024$ & $32-512$ & $4-16$ \\
\hline & MBL & $7.67 \pm 1.3$ & $8.11 \pm 1.5$ & $25.64 \pm 1.6$ & $>1024$ & $128-1024$ & $2-8$ \\
\hline & Efflux & $6.81 \pm 1.8$ & $10.51 \pm 1.8$ & $24.57 \pm 1.1$ & $>1024$ & $64-512$ & $2-16$ \\
\hline
\end{tabular}

$\mathrm{NZ}=$ No Zone

\section{DISCUSSION}

Indiscriminate use of antibiotics, poor patient compliance and improper infection control practices has led to emergence of multi drug resistant strains which transfer resistance through plasmids and confer resistance to commonly used cephaloroporin antibiotics. Combination therapy has been reported to be beneficial for the treatment of infections which fail to respond to single drug therapy because of lacking of efficacy or rapid emergence of resistance (Deresinski, 2009; Kumar et al., 2010). Although there are several data on the inter action of antibacterial agents against gram-positive and gramnegative organisms (Deveci et al., 2012), there is no report on the interaction between ceftriaxone and sulbactam and disodium edetate. Ceftriaxone in combination with sulbactam and disodium edetate show a greater susceptibility against resistant organisms as combination of trio, acting by different mechanisms, is used for the treatment of MDR bacterial infections.

The FIC index is the most commonly used method to determine the interaction between antibacterial drugs. The significant synergy was obtained at ratio 2:1 of ceftriaxone and sulbactam, which enhanced with increasing the concentration of disodium edetate and maximum synergy was found at $10 \mathrm{mM}$ of disodium edetateA, suggesting synergistic activity of ceftriaxone + sulbactam + disodium edetate. This AAE was synergistic for both positive controls as well as selected clinical isolates positive with ESBL, MBL and efflux. Earlier it was demonstrated that ceftriaxone montherapy is ineffective in the treatment ESBL but when it was combined with sulbactam and disodium edetate synergy was enhanced significantly (Chaudhary et al., 2012c). Deveci et al. (2012) studied the combinations of sulbactam with ceftriaxone, ceftazidime_and gentamicin against $A$. baumannii and observed synergy among these. The synergistic interaction between ceftriaxone plus sulbactam with disodium edetate was also demonstrated in animal model where combination therapy resulted in faster recovery in animals (infected with pneumonia) treated with combination compared with mono therapy (Dwivedi et al., 2012). Moreover, the synergistic interaction between ceftriaxone plus sulbactam with disodium edetate was also proven through clinical trials in patients suffering from Lower Respiratory Tract Infections (LTRIs), Urinary Tract Infections (UTIs), skin and skin structure infections (SSSIs) and Bone and Joint Infections (BJIs) (Chaudhary and Payasi, 2013a; 2013b).

The synergistic activity of ceftriaxone plus sulbactam with disodium edetate was also reported in efflux positive isolates of E. coli and P. aeruginosa (Chaudhary et al., 2012a; Chaudhary and Payasi, 2012). In addition, TKC, broth dilution, agar diffusion studies also carried out against all clinical isolates and indicated synergy between the ceftriaxone and sulbactam in a ratio of $2: 1$ with disodium edetate. Earlier, the synergistic activity of ceftriaxone with moxifloxacin was studied and found to be synergistic (Zakaria et al., 2012).

\section{CONCLUSION}

The current study revealed that novel AAE a combination of ceftriaxone plus sulbactam with 
disodium edtate could be the effective solution against the infections caused by A. baumannii, E. coli, K. pneumoniae and $P$. aeruginosa microorganisms positive with ESBL, MBL and efflux rather than searching for new antibiotics for treatment of infections caused by these organisms.

\section{ACKNOWLEDGEMENT}

Researchers are thankful to Venus Medicine Research Centre, Werne, Germany and BFMC, Faridkot, Punjab, India for providing assistance to carry out this study.

\section{REFERENCES}

Acevedo, J.G., J. Fernandsez, M. Castro, O. García and C. Rodriguez de Lope et al., 2009. Current efficacy of recommended empirical antibiotic therapy in patients with cirrhosis and bacterial infection. J. Hepatol., 50: S5-S5. DOI: 10.1016/S01688278(09)60008-8

Chaudhary M., M. Sudaroli, S. Kumar and V. Krishnaraju, 2012a. Catering ESBL resistance challenge through strategic combination of Ceftriaxone, Sulbactam and Ethylenediaminetetraacetic Acid. Int. J. Drug Dev. Res., 4: 72-81.

Chaudhary, M. and A. Payasi, 2012. Ethylenediaminetetraacetic acid: A non antibiotic adjuvant enhancing Pseudomonas aeruginosa susceptibility. Afr. J. Microbiol. Res., 6: 6799-6804. DOI: 10.5897/AJMR12.1407,

Chaudhary, M. and A. Payasi, 2013a. A randomized, open-label, prospective, multicenter phase-III clinical trial of Elores in lower respiratory tract and urinary tract infections. J. Pharmacy Res., 6: 409414. DOI: 10.1016/j.jopr.2013.04.011

Chaudhary, M. and A. Payasi, 2013b. Clinical, microbial efficacy and tolerability of Elores, a novel antibiotic adjuvant entity in ESBL producing pathogens: Prospective randomized controlled clinical trial. J. Pharmacy Res., 7: 275-280. DOI: 10.1016/j.jopr.2013.04.017

Chaudhary, M., G.K. Naidu, S. kumar and A. Payasi, 2012b. Comparative antibacterial activity of a novel semisynthetic antibiotic: Etimicin sulphate and other aminoglycosides. World J. Microbiol. Biotechnol., 12: 3365-71. DOI: 10.1007/s11274012-1148-5
Chaudhary, M., S. Kumar and A. Payasi, 2012c. A novel approach to combat acquired multiple resistance in escherichia coli by using disodium edetate as efflux pump inhibitor. J. Microb. Biochem. Technol. 4: 126-130.

CLSI, 2011. Performance standards for antimicrobial susceptibility testing; Twenty-first informational supplement. Clinical and Laboratory Standards Institure.

Deresinski, S., 2009. Vancomycin in combination with other antibiotics for the treatment of serious methicillin-resistant Staphylococcus aureus infections. Clin. Infect. Dis., 49: 1072-1079. PMID: 19725789

Deveci, A., A.Y. Coban, O. Acicbe, E. Tanyel and G. Yaman et al., 2012. In vitro effects of sulbactam combinations with different antibiotic groups against clinical Acinetobacter baumannii isolates. J. Chemother., 24: 247-252. PMID: 23182043

Dutil, L., R. Irwin, R. Finley, L.K. Ng and B. Avery et al., 2010. Ceftiofur resistance in salmonella enterica serovar heidelberg from chicken meat and humans, Canada. Emerg. Infect. Dis., 16: 48-52. PMID: 20031042

Dwivedi, V.K., P. Kumar and M. Chaudhary, 2012. Comparative study of CSE 1034 and Ceftriaxone in pneumonia induced rat. Clin. Exp. Pharmacol., 2: 108-108. DOI: 10.4172/2161-1459.1000108

Howard, A., M.O. Donoghue, A. Feeney and R.D. Sleator, 2012. Acinetobacter baumannii: An emerging opportunistic pathogen. Virulence, 3: 243250. PMID: 22546906

Karthika, R.U., S. Rao, S. Sahoo, P. Shashikala and R. Kanungo et al., 2009. Phenotypic and genotypic assays for detecting the prevalence of metallo- $\beta$ lactamases in clinical isolates of Acinetobacter baumannii from a South Indian tertiary care hospital. J. Med. Microbiol., 58: 430-435. PMID: 19273637

Khan, F., M. Rizvi, I. Shukla and A. Malik, 2011. A novel approach for identification of members of Enterobacteriaceae isolated from clinical samples. Biol. Med., 3: 313-319.

Kumar, A., N. Safdar, S. Kethireddy and D. Chateau, 2010. A survival benefit of combination antibiotic therapy for serious infections associated with sepsis and septic shock is contingent only on the risk of death: A meta-analytic/meta-regression study. Crit. Care Med., 38: 1651-1664. PMID: 20562695 
Lopes, B.S. and S.G.B. Amyes, 2013. Insertion sequence disruption of adeR and ciprofloxacin resistance caused by efflux pumps and gyrA and parC mutations in Acinetobacter baumannii. Int. J. Antimicrob. Agents, 41: 117-121. PMID: 23217848

Memish, Z.A., A.M. Shib, A.M. Kambal, Y.A. Ohaly and A. Ishaq et al., 2012. Antimicrobial resistance among non-fermenting gram-negative bacteria in Saudi Arabia. J. Antimicrob. Chemother., 67: 17015. PMID: 22461312

Muthusamy, D. and A. Boppe, 2012. Phenotypic methods for the detection of various $\beta$-lactamases in carbapenem resistant isolates of Acinetobacter baumanii at a tertiary care hospital in South India. J. Clin. Diag. Res., 6: 970-973.

Ohnishi, M., T. Saika, S. Hoshina, K. Iwasaku and S. Nakayama, 2011. Ceftriaxone- resistant Neisseria gonorrhoeae, Japan [letter]. Emerg. Infect. Dis., 17: 148-149. PMID: 21192886

Rawat, D. and D. Nair, 2010. Extended-spectrum $\beta$ lactamases in Gram Negative bacteria. J. Glob. Infect. Dis., 2: 263-274. PMID: 20927289

Tam, V.H., K.T. Chang, K. Abdelraouf, C.G. Brioso and M. Ameka et al., 2010. Prevalence, resistance mechanisms and susceptibility of multidrug-resistant bloodstream isolates of Pseudomonas aeruginosa. Antimicrob. Agents Chemother., 45: 1160-1164. PMID: 20086165
Unemo, M., D. Golparian, A. Hestner, 2010. Ceftriaxone treatment failure of pharyngeal gonorrhoea verified by international recommendations, Sweden. Euro. Surveill., 16: 19792-19792. PMID: 21329645

Wijayanti, M.A., E.N. Sholikhah and R.R. Hadanu, 2010. Additive in vitro antiplasmodial effect of $\mathrm{N}$ alkyl and N-benzyl-1,10-phenanthroline derivatives and cysteine protease inhibitor e64. Malaria Res. Treat., 2010: 540786-540786. PMID: 22332022

Yong, D., K. Lee, J.H. Yum, H. B. Shin and G.M. Rossolini et al., 2002. Imipenem-EDTA disk method for differentiation of metallo-betalactamase-producing clinical isolates of Pseudomonas spp. and Acinetobacter spp. J. Clin. Microbiol., 40: 3798-801. PMID: 12354884

Zakaria, A.S., N.A. Melake, N.A. Baky, N.M.E. Rasheed and N.H. Ibrahim, 2012. In vitro and in vivo studies of antibacterial effect of ceftriaxone moxifloxacin combination against methicillin resistant Staphylococcus aureus biofilms formed on biomedical implants. Afr. J. Microbiol. Res., 6: 5399-5409.

Zavascki, A.P., C.G. Carvalhaes, R.C. Picao and A.C. Gales, 2010. Multidrug-resistant Pseudomonas aeruginosa and Acinetobacter baumannii: Resistance mechanisms and implications for therapy. Exp. Rev. Anti. Infect. Ther., 8: 71-93. PMID: 20014903 